\title{
NOTE ON THE ESCALATOR METHOD
}

\section{BERNARD VINOGRADE}

Practical solution of the characteristic vector problem

$$
A X=\lambda X,
$$

where $A=\left(a_{i j}\right)$ is a given real nonsingular $n$-rowed matrix and $X$ is a column $\left(x_{1}, \cdots, x_{n}\right)^{\prime}$, has recently been treated anew by J. Morris. ${ }^{1}$ His process is called the escalator method. If $A_{p}$ is a $p$-rowed principal minor array in the upper left corner of $A$, then the abbreviated system

$$
A_{p} X_{p}=\lambda X_{p}, \quad p \leqq n,
$$

is called the $p$ th stage of (1). As given by Morris, the escalator method is based on expressions for the solutions of the $(p+1)$ th stage in terms of those of the pth stage, and depends on the existence of biorthonormal sets of solution vectors for (2) and the transposed system

$$
A_{p}^{\prime} Y_{p}=\lambda Y_{p} .
$$

But, of course, it is not always possible to find such biorthonormal sets of solution vectors, since the elementary divisors of the $p$ th stage may not be linear. In this note a more general exposition of escalation is given, covering all cases and leading to at least one practical method in cases outside the scope of Morris' formulas. In addition, a result on the transmission of roots from one stage to the next is included.

Let $\lambda_{i}$ be a characteristic root of multiplicity $n_{i}$ of the system (2), and $X_{p i}$ a corresponding characteristic vector. As is well known, there exists a nonsingular $P_{0}$ such that

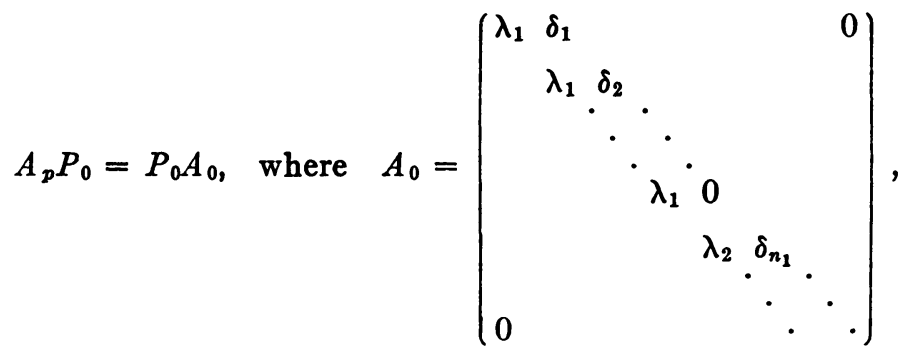

Received by the editors December 27, 1948.

${ }^{1} \mathrm{~J}$. Morris, The escalator method, Wiley, 1947. 
the $\delta_{i}$ 's being zero or one. If we set

$$
\begin{gathered}
C=\left(a_{p+1,1}, \cdots, a_{p+1, p}\right), \quad D=\left(a_{1, p+1}, \cdots, a_{p, p+1}\right)^{\prime}, \\
A_{p+1}=\left(\begin{array}{lc}
A_{p} & D \\
C & a_{p+1, p+1}
\end{array}\right), \quad P=\left(\begin{array}{cc}
P_{0} & 0 \\
0 & 1
\end{array}\right), \quad B=P^{-1} A_{p+1} P,
\end{gathered}
$$

then one can easily expand the equation

$$
\left|A_{p+1}-\lambda I_{p+1}\right|=\left|B-\lambda I_{p+1}\right|=\left|\begin{array}{cc}
A_{0}-\lambda I_{p} & P_{0}^{-1} D \\
C P_{0} & a_{p+1, p+1}-\lambda
\end{array}\right|=0,
$$

where $I_{p}$ is the $p$-rowed identity matrix. This equation is the general escalator equation for the determination of the characteristic roots at the $(p+1)$ th stage. When $A_{0}$ is diagonal, then (6) coincides with Morris' equation ${ }^{2}$ and is especially effective in symmetric systems. Since $A_{p}^{\prime} P_{0}^{-1 \prime}=P_{0}^{-1 \prime} A_{0}^{\prime}$, the transposed system (3) leads to the same escalator equation $\left|B^{\prime}-\lambda I_{p+1}\right|=0$.

For a determination of $P_{0}$, one may place $X_{p 1}$ in the first column, $X_{p 2}$ in the $\left(n_{2}+1\right)$ th column, and so on. The remaining coefficients and the $\delta_{i}$ 's may then be solved from the system (4). ${ }^{3}$

For a determination of the characteristic vectors of the $(p+1)$ th stage, ${ }^{4}$ let $X^{*}$ be a $p+1$-columned matrix of the unknown vectors and let $\Lambda$ be the diagonal matrix of corresponding characteristic roots of the $(p+1)$ th stage as determined from the escalator equation (6). Then from $A_{p+1} X^{*}=X^{*} \Lambda$ it follows that $P^{-1} A_{p+1} P P^{-1} X^{*}$ $=P^{-1} X^{*} \Lambda$, or $B Z^{*}=Z^{*} \Lambda$, where $Z^{*}=P^{-1} X^{*}$ and $B=P^{-1} A_{p+1} P$ (as defined by (5)). Because of the simple structure of $B$, the equations $B Z^{*}=Z^{*} \Lambda$ afford an easy determination of $Z^{*}$, hence of $X^{*}=P Z^{*}$.

As for the transposed system $A_{p+1}^{\prime} Y^{*}=Y^{*} \Lambda$, we note that if $Z^{*}$ is nonsingular (which is possible whenever the elementary divisors at the $(p+1)$ th stage are linear), it follows from $B Z^{*}=Z^{*} \Lambda$ that $B^{\prime} Z^{*-1^{\prime}}$ $=Z^{*-1^{\prime}} \Lambda$, hence $Y^{*}=P^{-1^{\prime}} Z^{*-1^{\prime}}=X^{*-1^{\prime}}$ is a solution of the transposed system.

If $C P_{0}=\left(P_{1}, \cdots, P_{p}\right), P_{0}^{-1} D=\left(Q_{1}, \cdots, Q_{p}\right)^{\prime}$, then a necessary and sufficient condition for a root $\lambda_{r}$ of the $p$ th stage to be a root of the $(p+1)$ th stage is $P_{r} Q_{r}=0$ for any pair $P_{r}, Q_{r}$ arising from a column of $P_{0}$ which is a characteristic vector of the $p$ th stage cor-

2 The components of $C P_{0}$ and $P_{0}^{-1} D$ are Morris' $P_{i}^{\prime}$ 's and $P_{i}^{\prime}$ 's.

${ }^{3}$ In connection with (4), the escalator method for simultaneous linear systems as compactly presented by R. A. Frazer, Note on the escalator method, Philosophical Magazine vol. 38 (1947) pp. 287-289, is pertinent.

4 Checks and other special procedures have been developed by R. J. Lambert, Master's Thesis, Iowa State College of Agriculture and Mechanic Arts, 1948. 
responding to $\lambda_{r}$.

For the necessity, let $x_{p+1}$ be the last component of $X_{p+1}$ and notice that the systems $A_{p+1} X_{p+1}=\lambda X_{p+1}$ and $A_{p+1}^{\prime} Y_{p+1}=\lambda Y_{p+1}$ may be written

$$
\begin{aligned}
\left(A_{p}-\lambda I_{p}\right) X_{p}+D x_{p+1} & =0 \\
C X_{p}+x_{p+1}\left(a_{p+1, p+1}-\lambda\right) & =0 \\
\left(A_{p}^{\prime}-\lambda I_{p}\right) Y_{p}+C^{\prime} y_{p+1} & =0 \\
D^{\prime} Y_{p}+y_{p+1}\left(a_{p+1, p+1}-\lambda\right) & =0 .
\end{aligned}
$$

Hence if $P_{r}=C X_{p r}=0$, then (7) is satisfied by $\lambda=\lambda_{r}$ and $X_{p+1}$ $=\left(X_{p r}^{\prime}, 0\right)^{\prime}$. Similarly, $Q_{r}=0$ implies $(8)$ is satisfied by $\lambda=\lambda_{r}$ and $Y_{p+1}=\left(Y_{p r}^{\prime}, 0\right)^{\prime}$.

On the other hand, if we left-multiply the $p$ equations $\left(A_{p}, D\right) X_{p+1}$ $=\lambda X_{p}$ by $Y_{p r}^{\prime}$ and recall that $A_{p}^{\prime} Y_{p r}=\lambda_{r} Y_{p r}$, then

$$
\left(\lambda_{r}-\lambda\right) Y_{p r}^{\prime} X_{p}+Q_{r} x_{p+1}=0 .
$$

Now if (9) is satisfied by $\lambda=\lambda_{r}$ and a corresponding vector $X_{p+1, r}$, then either $Q_{r}=0$ or $x_{p+1}=0$. If $x_{p+1}=0$, then the first $p$ components of $X_{p+1, r}$ satisfy the $p$ th stage, hence $P_{r}=0$. Thus the sufficiency is proved.

Iowa State College of Agriculture and Mechanic Arts 